\title{
ORT.05 - High Proteasome Activity in Plasma of Patients with Hematologic Malignancy (Case of I45 Moroccan patients)
}

\author{
Filali $\operatorname{Hassan}^{1 \star}$. \\ 1FST Mohammedia - Morocco.
}

Introduction: Present both in the cytoplasm and nucleus of all eukaryotic cells, the $20 \mathrm{~S}$ proteasome can be detected in peripheral blood (serum or plasma) (Stoebner and al, 2005). The proteasome, proteolytic heart of "ubiquitin-proteasome pathway" has a very broad substrate spectrum, most play a role in: the cell cycle, DNA repair, apoptosis (p53 and Caspase) angiogenesis (VEGF), inflammation (NF-kB, IL6 ....) immune response (antigen presentation) (Adams, 2002).

Objective: This study focused on a study in a large cohort of patients with Moroccan Hematologic malignancies in order to follow the evolution of the $20 \mathrm{~S}$ proteasome in serum and intracellular according to clinical status.

Methodology: Quantitative and functional analysis of the proteasome was conducted at the subcellular level and serum during a pathological phenomenon (hematologic malignancy) in 145 Moroccan patients (sex ratio: 1.10 / average age: $47.9 \pm 15.3$ years) with ELISA assay, and by following the fluorescence emitted after enzymatic digestion of specific peptides by the chymotrypsin-like activity.

Results: The evolutionary trend of subcellular proteasome is significantly linked to the rate of chymotrypsin-like activity, the entire population of 60 patients called back for a second blood test after three months of treatment reported a significant drop in the rate and the activity of the proteasome in serum and intracellular level.

Conclusion: The use of proteasome circulating assay as a biomarker of tumor and a tool that could be very satisfying to follow patients after remission to prevent a possible fall. So Intracellular dosage of proteasome reveals important because it allows estimating the predictive score of the risk of toxicity.

Keywords: Proteasome ; ELISA ; CTL-activity 